\title{
Vancomycin-resistant enterococci in Saudi Arabia: prevalence, antibiotic resistance and susceptibility array
}

\author{
Ayman Khalid Johargy ${ }^{1}$, Adil Jamal $2^{2 *}$ Aiman Mohamed Momenah ${ }^{1}$ and \\ Sami Sadagah Ashgar ${ }^{1}$ \\ 1. Department of Medical Microbiology, Faculty of Medicine, Umm Al-Qura University, Makkah, Saudi Arabia \\ 2. Molecular Biology, Department of Nursing Science and Research, College of Nursing, Umm Al-Qura University, \\ Makkah, Saudi Arabia \\ *Corresponding author's email: adiljamalcemb@gmail.com
}

Citation

Ayman Khalid Johargy, Adil Jamal, Aiman Mohamed Momenah and Sami Sadagah Ashgar. Vancomycin-resistant enterococci in Saudi Arabia: prevalence, antibiotic resistance and susceptibility array. Pure and Applied Biology. http://dx.doi.org/10.19045/bspab.2016.50104

\begin{tabular}{llll}
\hline \hline Received: 13/06/2016 & Revised: 01/08/2016 & Accepted: 04/08/2016 & Online First: 08/08/2016 \\
\hline \hline
\end{tabular}

\section{Abstract}

Vancomycin-resistant Enterococci (VRE) are an important cause of nosocomial infection and may result in increased morbidity and mortality in immunocompromised populations particularly in developing contries. The aim of the present study was to reassess the definition of hospitalacquired Enterococci, with particular emphasis on the relevance of recent hospitalization.Total 228 Enterococci clinical samples were collected from different hospitals of Saudi Arabia. Sampling was performed following sub culturing, strains isolation and antimicrobial susceptibility testing. Out of 228 Enterococci clinical isolates isolated in this study, 186 (82\%) were susceptible to vancomycin, $17(7 \%)$ were resistant and $25(11 \%)$ were intermediate. Most of VRE isolated in our study were from Jeddah hospitals $11 \%$. The most common infections caused by Enterococci in current study showed urinary tract infection (56.6\%) followed by blood (12.3\%) and wound infection (11.4\%). Porportion of $87 \%$ and $13 \%$ Enterococci isolated in this study were from Saudi and non-Saudi patients respectively and were calculated statistically nonsignificant ( $\mathrm{p}$-value $=0.4$ ). This limited study exhibited a high pervasiveness rate of multiple antibiotic resistant Enterococci infections among hospitalized patients in this environment. There is need for precise observations of clinics and hospitals for Enterococci infections; prudent use and rational prescription of antimicrobials and stringent measures to reduce the prevalence rate by health education on infection control measures such as isolation, cleaning, disinfection and sterilization.

Key words: Enterococci; Vancomycin; Resistance; Susceptibility; Saudi Arabia

Introduction

Enterococci are hardy, facultative anaerobic Gram positive cocci in pairs or short chains that are capable to grow and survive in many environments [1]. They are part of normal intestinal flora of humans and animals but may be responsible for serious infections. Of the 20 Enterococcus species [2], 2 species are particularly pathogenic to man; Enterococcus faecalis causes $85-90 \%$ of Enterococci infections while Enterococcus faecium causes 5-10\% [3]. 
Enterococci are among the most frequent causes of nosocomial infection, particularly in intensive care units (ICU) where they are selected by treatment with cephalosporin and other antibiotics to which they are resistant. They are transmitted from individual to individual primarily on the hand of hospital personnel (hospital acquired), some of whom may carry the organism in their gastrointestinal tracts. Less frequently, Enterococci are transmitted on medical devices. Meningitis and bacteraemia while endocarditis may occur in neonates and adults respectively. Enterococci infection is equally distributed between sexes, although urinary tract infections (UTI) are more common in healthy women than men and in elderly patients due to high incidence of urinary instrumentation [4].

Hospital-acquired (HA) infective endocarditis (IE) constitutes 9\%-29\% of all cases of IE [5] and has expanded in frequency in recent years to more prominent utilization of intrusive strategies in clinics. Community-acquired (CA) infections due to Enterococci are on ascent because of more utilization of broad spectrum antimicrobials. Moreover, since VRE from animal sources such as poultry and human foods of animal origin assume critical part role in human colonization and infection, a huge level of VRE colonization may be found among persons not linked with the health care setting [6].

VRE are basic reason for nosocomial infection and might bring about expanded dreariness and mortality in immunocompromised populaces. Enterococci accounted $14.7 \%$ and $7.1 \%$ of UTI and blood infection respectively. Enterococci clinical isolates indicate less assorted qualities besides those found in earth and human based sources, among $E$. faecalis most prevalent and overwhelming species. The explanation behind this absence of differing qualities might be connected with the harmfulness elements connected with this species [7]. Enterococci though are resistant to antibiotic, although a few species (e.g., E. faecium) are more characteristically safe than others. Low partiality tying protein expression triggers as a result of diminished helplessness to penicillin and ampicillin as huge imperviousness to most cephalosporin anti-infection agents [8].

Trimethoprim-sulfamethoxazole is proposed for dermatitis and delicate tissue infections brought about by Enterococci [9]. Abnormal state aminoglycoside resistance, ordinarily intervened by aminoglycoside-altering proteins, has been found in Enterococcal isolates [10]. Imperviousness to fluoroquinolones, in abundance of the humble inborn imperviousness found over numerous Enterococci, exists after effect of modifications in compounds included in reduplication of DNA [11]. Further basic procured hereditary cognitive factors present imperviousness to macrolides, tetracycline and chloramphenicol.Chromosomal transformations might deliver imperviousness to rifampin (rifampicin) and fusidic corrosive. Diverse sorts of VRE have been depicted on genetic and appearance bases [12].

Clinical disengages of Enterococci ought to be screened for vancomycin resistance. Agar screening plates provide straightforward, simple test for vancomycin resistance and are prescribed by the national board of trustees for clinical research facility gauges [13-14]. Imperviousness to direct and significant groupings of vancomycin is effectively identified by standard powerlessness testing strategies.

\section{Objective}

The aim of the present study was to reassess the definition of hospital-acquired Enterococci, with particular emphasis on the relevance of recent hospitalization.

\section{Materials and methods}

Samples collection 
A total number of 228 Enterococci clinical isolates were collected in this study from different hospitals of Saudi Arabia. Sampling was performed following urine, wound, high vaginal swab (HVS), bone, blood, eyes, abdominal and placental swabs, pleural fluid, cerebrospinal fluid (CSF), sputum, stool and fetal part.

Sub culturing of specimens and isolation of strains

Specimens were sub cultured on blood and bile esculin agar under complete aseptic conditions and incubated for $24 \mathrm{hrs}$ at $37^{\circ} \mathrm{C}$ to grow single pure colonies for performing gram stain and catalase test. All isolated bacteria were preserved at $-80^{\circ} \mathrm{C}$ in an eppendorf tubes containing $1 \mathrm{ml}$ brain heart infusion broth along $16 \%$ glycerol.

Identification of bacteria and antimicrobial susceptibility testing (AST)

Preserved bacteria were sub cultured in a suitable media and incubated accordingly to obtain pure culture. Ampicillin, gentamycin, vancomycin, tetracycline and ciprofloxacin were used for susceptibility testing. Around $3 \mathrm{ml}$ of clean saline (fluid $0.45 \% \mathrm{NaCl}, \mathrm{pH}$ 7) was put into a reasonable plastic test tube. By then adequate number of morphologically comparative states of immaculate microbes was exchanged to a tube containing the saline to make homogenous suspension with an identical thickness of McFarland (No.0.50-0.63) using calibrated VITEK2 DENSICHEK. The tube was then put in the tape with the recognizable proof card and information passage. To another tube containing $3 \mathrm{ml}$ of saline, $280 \mathrm{ml}$ of the suspension prepared for AST-GP was then transferred. The tube was then put in the anti-infection agents weakness tape, the recognizable proof GP depends on 43 biochemical tests measured carbon source usage, enzymatic actions and resistance. For the minimum inhibitory concentration (MIC) technique, AST card contains 64 micro wells were used. While control well was occupant on all card with the remaining wells containing premeasured measures of particular antimicrobials consolidated with culture medium. MIC values were determined for each antimicrobial contained on the card after a defined period of time (about $18 \mathrm{hrs}$ ).

\section{Data analysis}

Data was statistically analyzed in Microsoft Excel (ver 2010) by p values. Data was tabulated using a Fisher test (Graph Pad Instat program statistical software). P-values computed $<0.05$ were considered as significant.

\section{Results}

A total of 228 Enterococci clinical isolates were isolated in this study and tested for vancomycin susceptibility. In this study 186 (82\%), 17 (7\%) and $25(11 \%)$ Enterococci isolates were found to be susceptible, resistant and intermediate respectively. Clearly, Enterococci were isolated mostly from urine (129 isolates), blood (28 isolates) and wound samples (26 isolates). This indicates that the common infections caused by Enterococci in our study were mainly UTI followed by blood stream and wound infection (Figure 1). Most VRE isolated in our study were from Jeddah (11\%) followed by Makkah (9\%) and Taif hospitals (3\%) (Figure 2). 


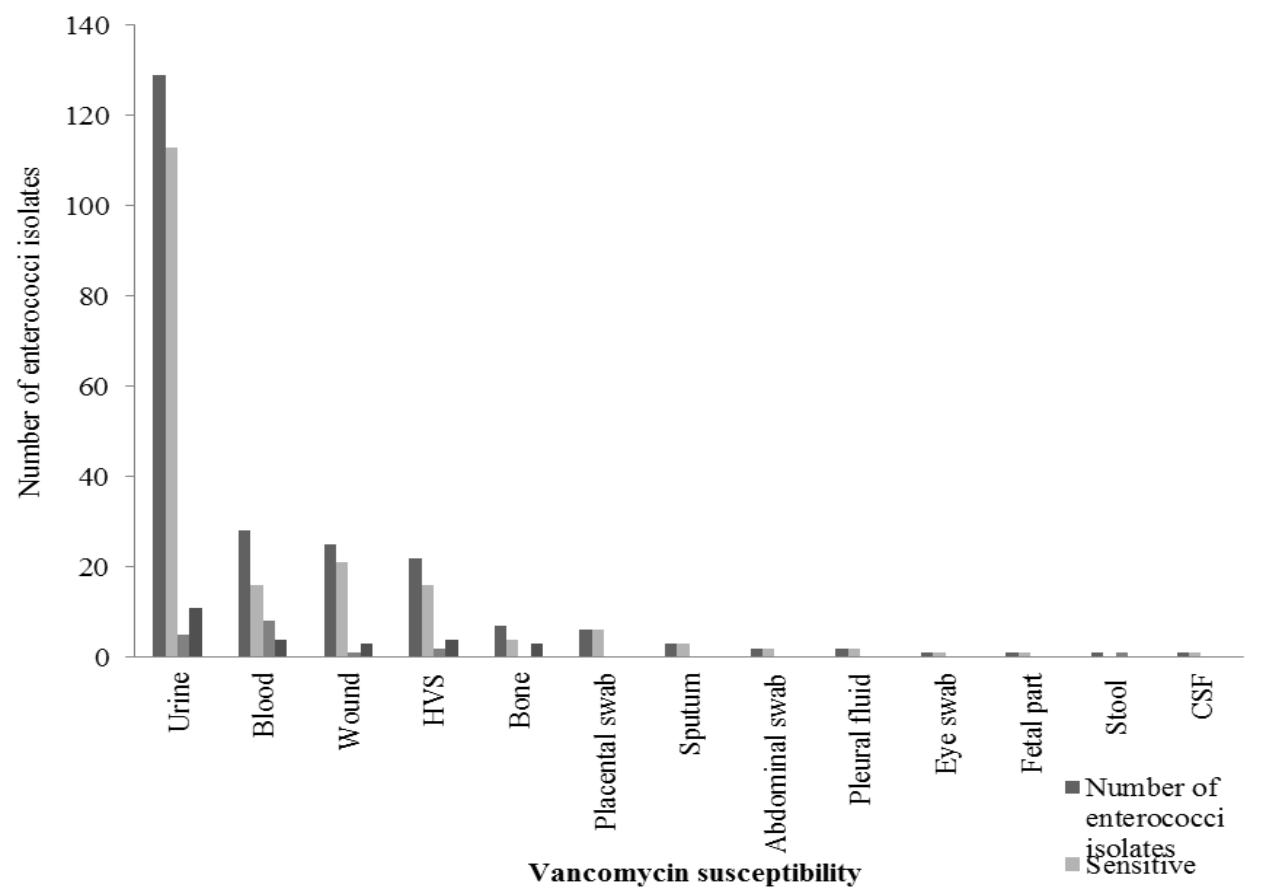

Figure 1. Distribution of Vancomycin resistant enterococci (VRE) isolates from different samples

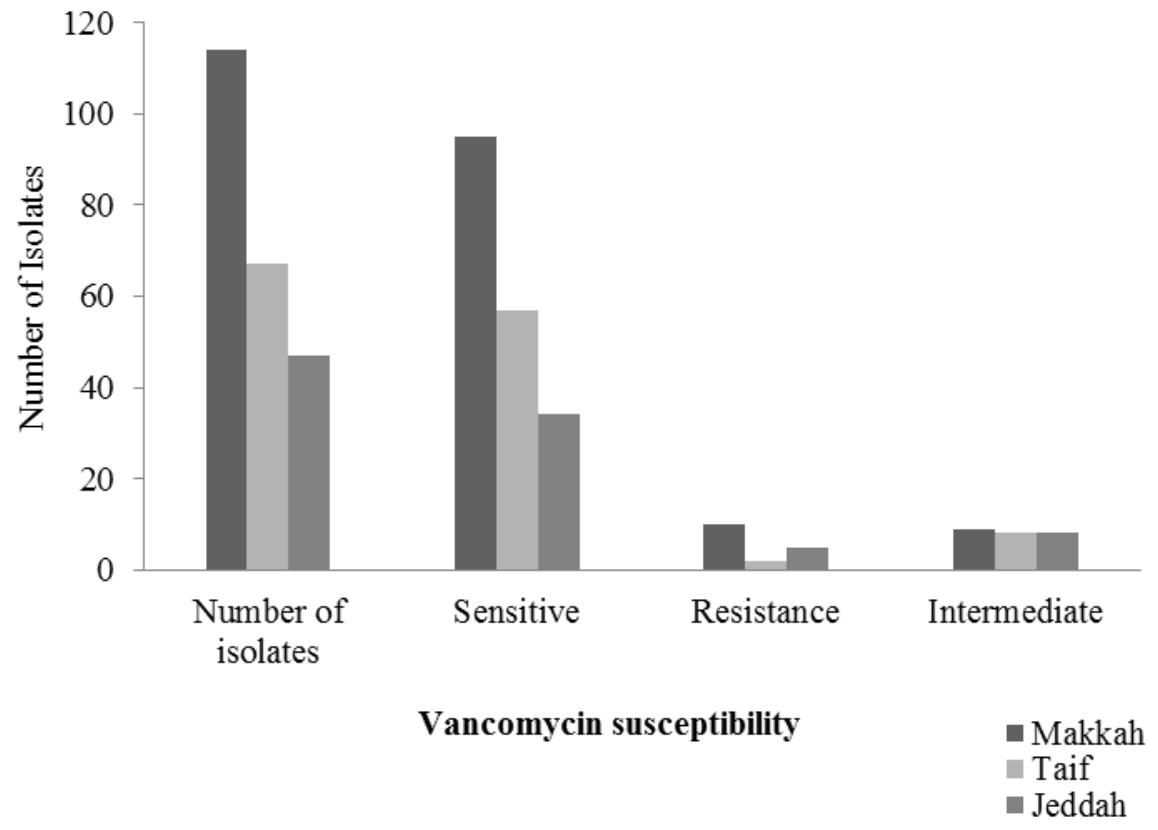

\section{Figure 2. Distribution of VRE isolates from different cities}

Data regarding the VRE isolates from different wards of hospital displayed the higher number of Enterococci (41 isolates) from intensive care unit (ICU), 40 from female medical ward (FMW), 32 from male medical ward (MMW), 18 from surgery, 16 from pediatric, 8 from orthopedic, 5 from nephrology, 4 from hemodialysis, 3 from OBS/Gyn and 1 from antenatal wards. 100\% Enterococci isolates were found to be 
sensitive collected from Nephrology, the ICU and pediatric wards exhibited 12\% OBS/Gyn, antenatal wards followed by Enterococci isolates followed by $9.4 \%$ in FMW, surgery, ICU, MMW, hemodialysis, $\quad$ MMW and 5\% in FMW (Figure 3). pediatric and orthopedic ward, while both

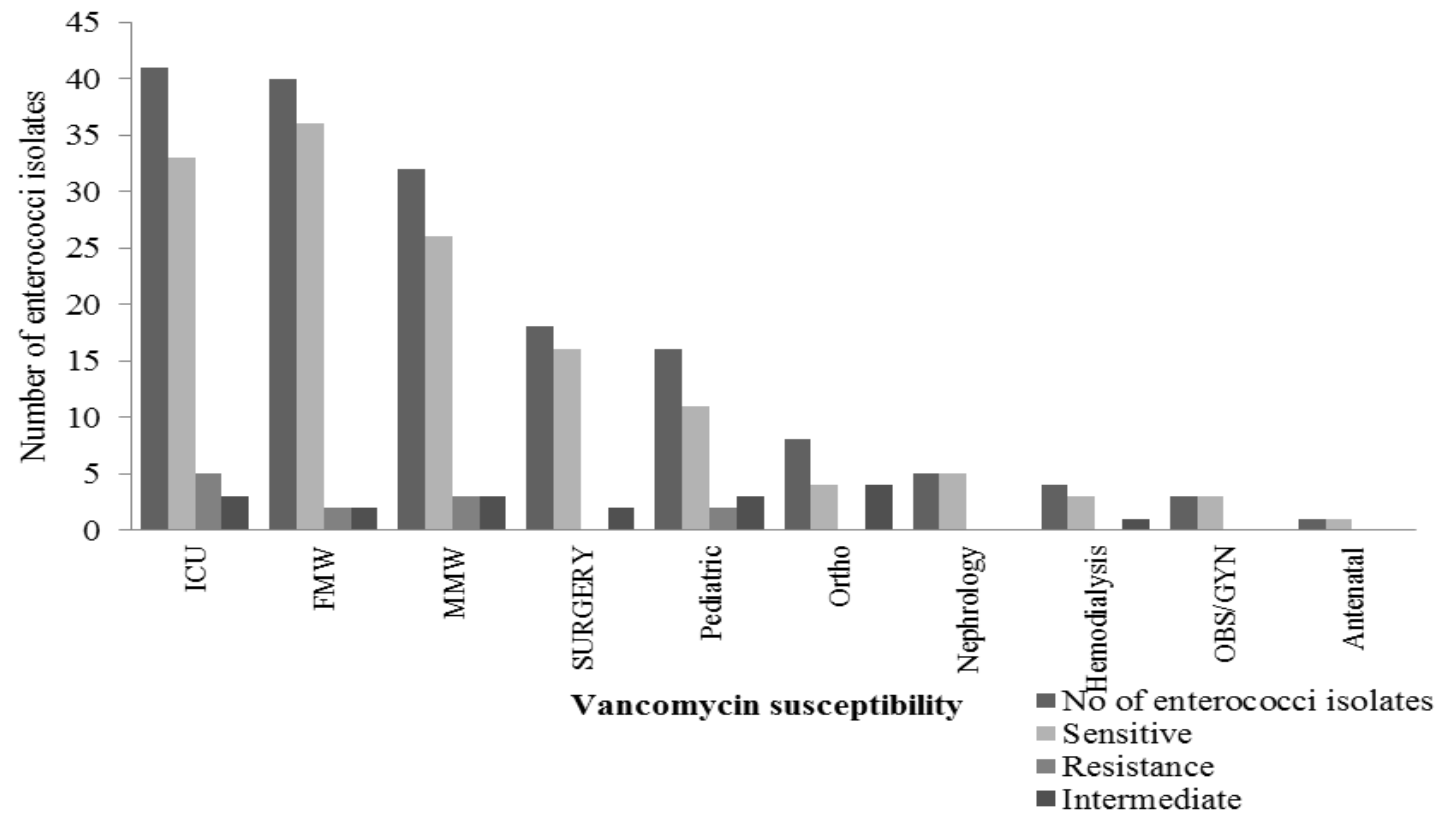

Figure 3. Distribution of VRE isolates among different hospitals wards

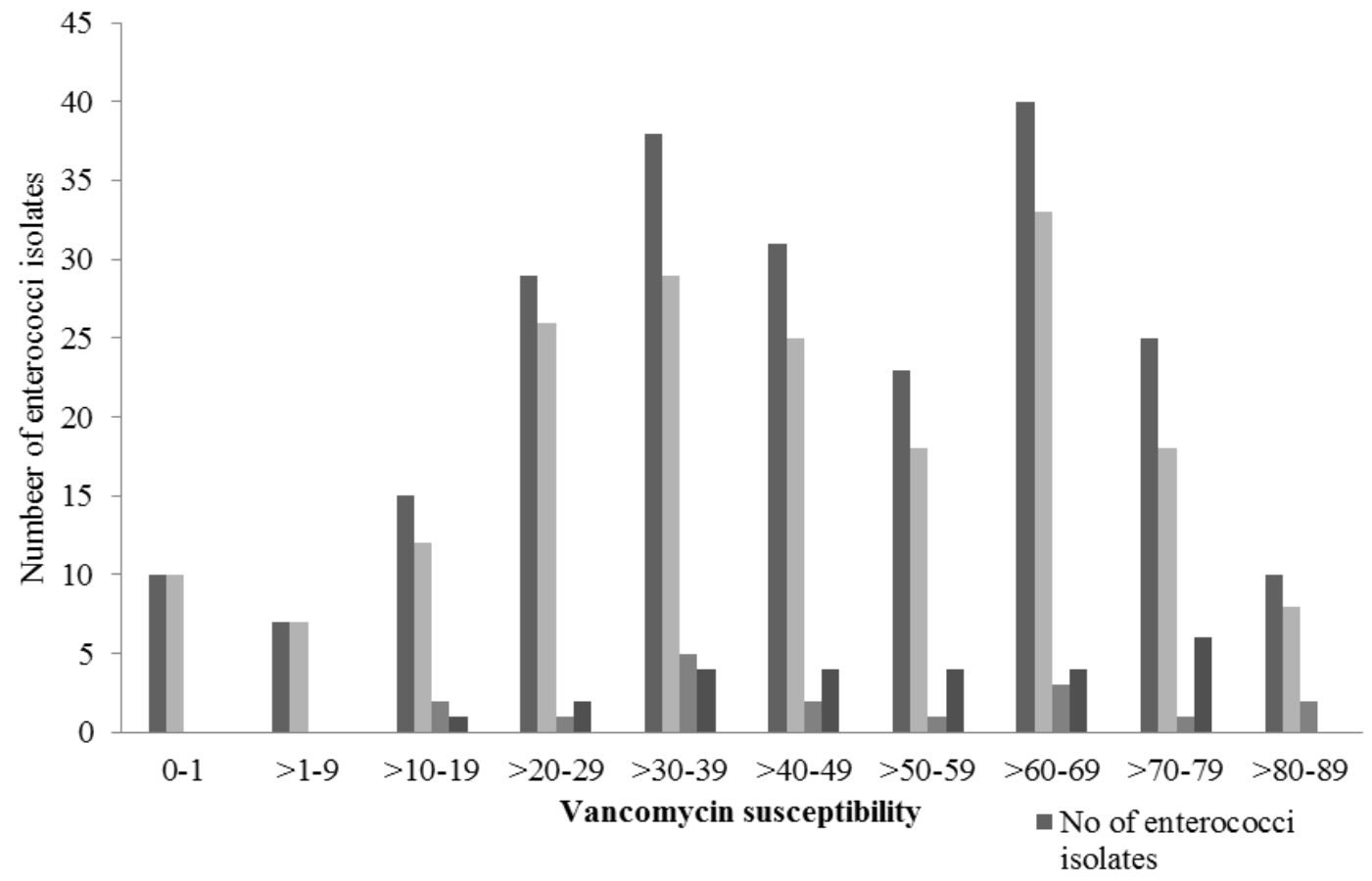

Figure 4. Distribution of VRE isolates among age groups 
Current data deduced that out of the 228 Enterococci isolates in this study, 95 were from male patients and $11(12 \%)$ of them were VRE, while 133 were from female patients and $6(4.5 \%)$ of them were VRE, however the mean difference in VRE between genders was quiet statistically nonsignificant $(\mathrm{p}$-value $=0.07)$. Out of the 228 Enterococci isolated in this study, 204 were from Saudi patients and $14(7 \%)$ of them were VRE, while 24 were from non-Saudi patients and $3(13 \%)$ of them were VRE. Regarding nationalities statistically non- significant difference was reported (pvalue $=0.4$ ). About $58.8 \%$ and $41.2 \%$ of Enteroccoccal infections in our study accounted for age above 50 and less than 50 years respectively. Difference in VRE between age groups was found statistically non-significant ( $\mathrm{p}$-value $=1)$ (Figure 4).

Regarding AST it was concluded that $69.75 \%, 48.2 \%, 42.6 \%, 66.6 \%$ and $7 \%$ of the Enterococci isolates were found resistant to ampicillin, gentamycin, ciprofloxacin, tetracycline and vancomycin respectively (Figure 5).

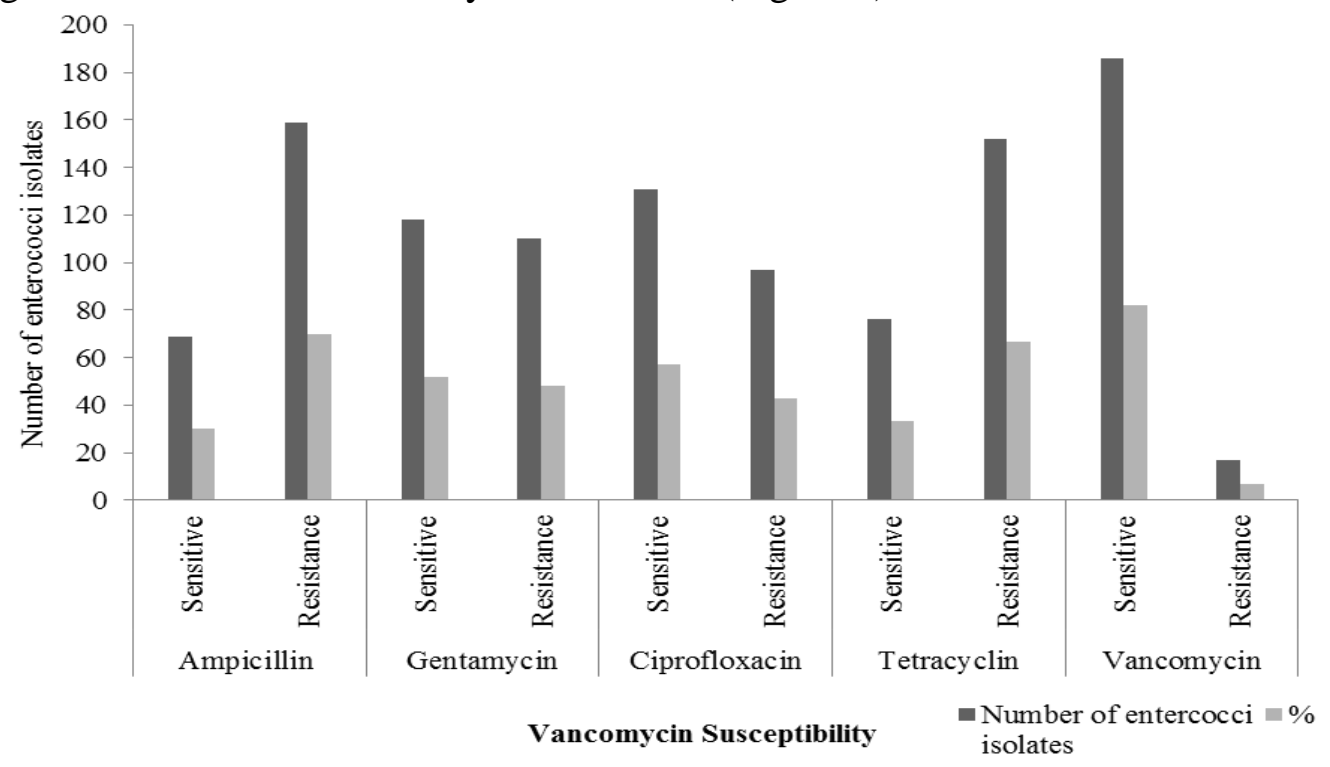

Figure 5. VRE Antibiotics susceptibility

\section{Discussion}

Hazard factors for VRE colonization consist of host attributes (immunosuppression, neutropenia, and renal deficiency) [15], hospice factors (ICU area or oncology ward, vicinity to a VRE-colonized patient and extended length of stay) [16-17] and antimicrobial application [18]. VRE colonization and relocation independently increases a patient's risk of developing infections, such as bloodstream infections (BSIs) [19]. VRE, BSI is generally linked with the belated administration of ample antimicrobials. The classification of risk factors and outcomes of VRE, BSI is critical for establishing strategies for managing neutropenic fever in patients with hematological malignancies [20]. It has been demonstrated that vancomycin resistance increases mortality in patients with Enterococcal BSI [21-22]; however, controversy remains [15, 23-24].

$E$ faecalis and $E$ faecium are generally viewed as life forms of moderately low destructiveness, in spite of the fact that intrusion can bring about UTI, meningitis and intra-stomach diseases. Antimicrobial drugs resistance is a developing general health issue and multidrug-resistant (MDR) pathogens such as VRE are increasing 
worldwide [25]. The pervasiveness of VRE in our study was $7 \%$ as compared to the previous reports in neighboring country [26]. The most common infections caused by Enterococci in our study were UTI $(56.6 \%)$ followed by BSI $(12.3 \%)$ and wound infection (11.4\%). Present results strongly supported by previous findings [27]. Literature also deduces that blood was the most common infections caused by Enterococci followed by UTI and wound infections [28]. Most of VRE infections found in our study were isolated from pediatric wards $(12.5 \%)$ followed by ICU $(12.3 \%)$. Highest rate of VRE occurred predominantly in ICU as supported by preceding conclusions [29].

In the current study, the mean age of Enterococci-infected patients ranged $44 \pm 21$ years. However, literature suggested mean age of Enterococci-infected patients ranged from $26.9 \pm 21.9$ years [30]. Present study showed proportion of $58.8 \%$ Enterococcal infections in patients with age less than 50 years as compared with $41.2 \%$ in patients above 50 years of age which is also supported by previous findings [31]. Enterococcal infections based upon gender differences in present study revealed proportion of $64.7 \%$ and $35.3 \%$ of from male and female patients respectively. Statistically non-significant difference was measured between genders. VRE infection affects more commonly male gender as that of female gender [32].

MDR becoming prominent worldwide among pathogen and abatement the choices for clinicians in the infections. MDR is a standout amongst the most critical normal for the Enterococci. High mortality rates of Enterococcal infections are because of the challenges included in the treatment of MDR pathogens [33]. Because of less effectiveness of dynamic transport over the cytoplasmic layer, Enterococci are characteristically impervious to low levels of aminoglycosides [34]. Aminoglycosides alone are viewed as latent in Enterococcal medication and are typically consolidated with cell inhibitors divider amalgamation which might encourage their further endorsement [35].

In current study, gentamycin expressed significant resistance against Enterococci. comparative congenital association of gentamicin alone beside two strains of $E$ faecalis was contemplated in vitro which fundamentally decreased the quantity of surviving microscopic organisms [36]. Our study shows that the characteristic action of gentamicin ought to be considered in concentrating on different aminoglycosides based medications and specialists dynamic against the cell divider, for example, ampicillin or vancomycin. Ciprofloxacin (fluoroquinolones) have an expansive range for gram-positive and gram-negative microscopic organisms. For UTI fluoroquinolones is recommended and standard medication [37].Besides, fluoroquinolones have been utilized partially or completely to treat E. faecalis related UTIs. Therefore, the rise of fluoroquinolonesafe $E$. faecalis strains has been accounted for few nations [38]. Our outcomes appeared even noteworthy affectability of ciprofloxacin against Enterococci which is firmly bolstered and prove by past results. Along these lines, Ciprofloxacin is no more endorsed treatment for E. faecalis based UTI [39].

Resistance to tetracycline is frequent in clinical isolates of Enterococci. The presence of strains resistant to tetracycline has also been described in diverse animal based food origin [40, 41]. In Enterococci, tetracycline resistance is generally associated to the presence of the gene tet(M) which confers ribosomal protection, but other related genes affording ribosomal protection [42]. 
In current study, tetracycline showed increased resistance level against Enterococci. Previous studies also reported poor activity of tetracycline against antimicrobials especially in periodontal pockets infected with E. faecalis and hence sometimes it seems to be ineffective therapeutic agent against E. faecalis [42]. Among orally managed anti-infection agents, ampicillin, amoxicillin and ciprofloxacin showed set apart in vitro inhibitory action against periodontal $E$. faecalis, and might be clinically valuable in treatment of periodontal diseases including Enterococci [42].

Current study concludes that antimicrobials based drugs like tetracycline articulated higher resistance against Enetrococcci followed by tetracycline, ciprofloxacin, gentamycin and vancomycin while increased activity of sensitivity was analyzed in antimicrobials viz; vancomycin followed by ciprofloxacin, gentamycin, tetracycline and ampicillin against Enterococci. Based upon the inference it is concluded that antimicrobial vancomycin has the higher sensitivity with least resistance against Enterococci. The role of these drugs according to their resistance and sensitivity levels can be manipulated and wrought by clinicians against Enterococci based infections in future.

\section{Conclusion}

Findings regarding prevalence and persistence of VRE among clinical isolates will help the clinician to estimate the extent of the problem of resistance among enterococci and ultimately help to design the appropriate clinical preventive measures.

\section{Acknowledgements}

The authors would appreciate to the students and Department of Medical Microbiology staff, Faculty of medicine, Umm Al Qura University, Makkah for their assistance during sample collection.

\section{Authors' contributions}

Planned, Designed, Developed and Approved study concept, Supervised in collection, Assembly, Possession of raw data, Funds arrangement: AK Johargy, Performed data interpretation, Statistical analysis, Critical discussion and Preparation of this manuscript: A Jamal, Sampling, administrative, technical and material support: AM Momenah \& SS Ashgar.

\section{Funding/ support}

Authors highly acknowledge the funding granted by the Medical Research Center of the Institute of the Scientific Research and Islamic Heritage Revival, Umm Al-Qura University, Makkah, Saudi Arabia to accomplish this research project.

Competing Interest: All authors have declared that they have no competing interest.

\section{References}

1. Murray BE (1990). The life and times of the enterococcus. Clin Microbiol Rev. 3:45-65.

2. Facklam RR, Carvalho MS \& Teixeira LM (2002). History, taxonomy, biochemical characteristics and antibiotic susceptibility testing of enterococci. In: Gilmore MS, Clewell DB, Courvalin P, Dunny GM, Murray BE, Rice LB, editors. The enterococci, pathogenesis, molecular biology and antibiotic resistance. Washington, DC: ASM Press; pp. 1-54.

3. Patterson JE, Sweeney AH, Simms M, Carley N, Mangi R, Sabetta J \& Lyons RW (1995). Analysis of 110 series enterococcal infections. Medicine. 74:191-200.

4. Gordon S, Swenson JM, Hill BC, Piggot NE, Facklam RR, Cooksey RC, Thornsberry C, Jarvis WR \& Tenover FC (1992). Antimicrobial susceptibility patterns of common and unusual species of enterococci causing infections in the 
United States. $J$ Clin Microbiol. 30:2373-2378.

5. Chen SC, Dwyer DE \& Sorrell TC (1993). A comparison of hospital and community-acquired infective endocarditis. Am J Cardiol 70:1449-52.

6. Mathur P \& Singh S (2013). Multidrug resistance in bacteria: A serious patient safety challenge for India. $J$ Lab Physicians. 5:5-10.

7. Kuhn I, Iversen A, Burman LG, OlssonLiljequist B, Franklin A and Finn M, et al (2003). Comparison of enterococcal populations in animals, humans and the environment - a European study. Int $J$ of Food Microbiol. 88(2-3): 133-145.

8. Kristich CJ, Rice LB \& Arias CA (2014). Enterococcal infection-treatment and antibiotic resistance. Enterococci: From commensals to leading causes of drug resistant infection. Boston: Massachusetts Eye and Ear Infirmary.

9. Paul M, Bishara J, Yahav D, Goldberg E, Neuberger A and Nesrin G-Z, et al (2015).Trimethoprim-sulfamethoxazole versus vancomycin for severe infections caused by meticillin-resistant Staphylococcus aureus: randomized controlled trial. BMJ. 350:h2219.

10. Adhikari L (2010). High-level Aminoglycoside resistance and reduced Susceptibility to vancomycin in nosocomial Enterococci. J Glob Infect Dis. 2(3): 231-235.

11. Jacoby GA (2005). Mechanisms of resistance to quinolones. Clin Infect Dis. 41 Supp 2: 120-126.

12. Praharaj I, Sujatha S \& Parija SC (2013). Phenotypic and genotypic characterization of vancomycin resistant Enterococcus isolates from clinical specimens. Indian J Med Res. 138(4): 549-556.

13. Jorgensen JH \& Ferraro MJ (2000). Antimicrobial susceptibility testing: special needs for fastidious organisms and difficult-to-detect resistance mechanisms. Clin Infect Dis. 30(5):799808.

14. Swenson JM, Patel JB \& Jorgensen JH (2011). Special phenotypic methods for detecting antibacterial resistance. In: Murray PR, Baron EJ, Jorgensen JH, Pfaller MA, Yolken RH, editors. $10^{\text {th }}$ ed. Washington DC: ASM Press. pp. 117895.

15. Lautenbach E, Bilker WB \& Brennan PJ (1999). Enterococcal bacteremia: risk factors for vancomycin resistance and predictors of mortality. Infect Control Hosp Epidemiol 20:318-23.

16. Bonten MJ, Slaughter S \& Ambergen AW et al (1998). The role of "colonization pressure" in the spread of vancomycin-resistant enterococci: an important infection-control variable. Arch Intern Med 158:1127-32.

17. Lucas GM, Lechtzin N, Puryear DW, Yau LL, Flexner CW \& Moore RD (1998). Vancomycin-resistant and vancomycin-susceptible enterococcal bacteremia: comparison of clinical features and outcomes. Clin Infect Dis 26:1127-33.

18. Edmond MB, Ober JF \& Weinbaum DL, et al (1995). Vancomycin-resistant Enterococcus faecium bacteremia: risk factors for infection. Clin Infect Dis 20:1126-33.

19. Roghmann MC, McCarter RJ, Brewrink J, Cross AS \& Morris JG (1997). Clostridium difficile infection is a risk factor for bacteremia due to vancomycin-resistant enterococci (VRE) in VRE-colonized patients with acute leukemia. Clin Infect Dis 25:1056-9.

20. Cho S-Y, Lee D-G, Choi S-M, Kwon JC, Kim S-H \& Choi J-K, et al (2013). Impact of vancomycin resistance on mortality in neutropenic patients with enterococcal bloodstream infection: a 
retrospective study. BMC Infectious Diseases 13:504.

21. DiazGranados CA, Zimmer SM, Klein M \& Jernigan JA (2005). Comparison of mortality associated with vancomycinresistant and vancomycin-susceptible enterococcal bloodstream infections: a meta-analysis. Clin Infect Dis. 41: 327333

22. DiazGranados CA \& Jernigan JA (2005). Impact of vancomycin resistance on mortality among patients with neutropenia and enterococcal bloodstream infection. J Infect Dis. 191: 588-595.

23. Garbutt JM, Ventrapragada M, Littenberg B \& Mundy LM (2000). Association between resistance to vancomycin and death in cases of Enterococcus faecium bacteremia. Clin Infect Dis. 30: 466-472.

24. Todeschini G, Tecchio C, Borghero C, D'Emilio A, Pegoraro E \& de Lalla F et al (2006). Association between Enterococcus bacteraemia and death in neutropenic patients with haematological malignancies. $J$ Infect. 53: 266-273.

25. GM E (2009). Microbiology of drugs for treating multiply drugresistant grampositive bacteria. J Infect. 59:Suppl 1: S17-24.

26. Sayed SBE, Nasr RA \& Shaheen MA (2005). Risk of Colonization of methicillin-resistant Staphylococcus aureus (MRSA) and vancomycinresistant Enterococci (VRE) in patients admitted to pediatric intensive care unit of Ain Shams University Hospitals. Egypt J Med Lab Sci, (ESIC). 14(2): 1-9.

27. Reika R, Tenoverb FC, Kleinc E \& McDonaldb LC (2008). The burden of vancomycin-resistant enterococcal infections in US hospitals, 2003 to 2004. Diagnostic Microbiol and Infect Dis. 62: 81-85.
28. Chatterjee I, Dulhunty JM, Iredell J, Gallagher JE, Sud A \& Woods M (2009). Predictors and outcome associated with an Enterococcus positive isolate during intensive care unit admission. Anaesth Intensive Care. 37(6):976-982.

29. Azevedo d' PA, Santiago KADS, Furtado GHC, Xavier DB, Pignatari ACC \& Titze-de-Almeida R (2009). Rapid detection of vancomycin-resistant Enterococci (VRE) in rectal samples from patients admitted to intensive care units. The Brazilian $J$ of Infect Dis. 13(4): 289-293.

30. Altoparlak U1, Koca O, Ozkurt Z \& Akcay MN (2011). Incidence and risk factors of vancomycin-resistant enterococcus colonization in burn unit patients. Burns. 37(1):49-53.

31. Weber SG, Miller RR, Perencevich EN, Tolentino J, Meltzer D, Pitrak D, McGregor JC, Sachs GA, Harris AD \& Furuno JP (2009). Prevalence of antimicrobial-resistant bacteria isolated from older versus younger hospitalized adults: results of a two-centre study. $J$ of Antimicrobial Chemo. 64: 1291-1298.

32. Abebe W, Endris M, Tiruneh M \& Moges F (2014). Prevalence of vancomycin resistant Enterococci and associated risk factors among clients with and without HIV in Northwest Ethiopia: a cross-sectional study. $B M C$ Public Health 14:185.

33. Leclercq R (1997). Enterococci acquire new kinds of resistance. Clin Infect Dis. 24 Suppl 1:S80-4.

34. Robert C, Moellering Jr \& Weinberg AN (1971). Studies on antibiotic syngerism against enterococci. II. Effect of various antibiotics on the uptake of 14 C-labeled streptomycin by enterococci. Weinberg AN J Clin Invest. 50(12):2580-4.

35. Lefort A, Arthur M, Garry L, Carbon C, Courvalin P \& Fantin B (2000). 
Bactericidal activity of gentamicin against Enterococcus faecalis In vitro and In vivo. Antimicrob Agents Chemother. 44(8):2077-80.

36. Lee G, Cho YH, Shim BS \& Lee SD (2010). Risk factors for antimicrobial resistance among the Escherichia coli strains isolated from korean patients with acute uncomplicated cystitis: a prospective and nationwide study. $J$ Korean Med Sci. 25(8):1205-9.

37. Matsumoto T, Hamasuna R, Ishikawa $K$, Takahashi S, Yasuda M \& Hayami H et al (2011). Nationwide survey of antibacterial activity against clinical isolates from urinary tract infections in Japan (2008). Int J Antimicrob Agents. $37(3): 210-8$.

38. Lee G (2013). Ciprofloxacin Resistance in Enterococcus faecalis Strains isolated from male patients with complicated urinary tract infection. Korean J Urol. 54(6): 388-393.

39. Butaye P, Van Damme K, Devriese LA, Van Damme L, Bael M \& Lauwers S et al (2000). In vitro susceptibility of Enterococcus faecium isolated from food to growth-promoting and therapeutic antibiotics. Int $J$ Food Microbiol. 54(3): 181-187.

40. Aarestrup FM, Agerso Y, Gerner-Smidt P, Madsen M \& Jensen LB (2000). Comparison of antimicrobial resistance phenotypes and resistance genes in Enterococcus faecalis and Enterococcus faecium from humans in the community, broilers and pigs in Denmark. Diagn Microbiol Infect Dis. 37(2): 127-37.

41. Pavia M, Nobile CG, Salpietro L \& Angelillo IF (2000).Vancomycin resistance and antibiotic susceptibility of Enterococci in raw meat. $J$ Food Prot. 63: 912-915.

42. Rams TE, Feik D, Mortensen JE, Degener JE \& Winkelhoff AJ (2013). Antibiotic Susceptibility of Periodontal Enterococcus faecalis. J Periodontol. 84(7):1026-33. 